\title{
High-Temperature Operation of Single-Electron Transistors Based on Single-Walled Carbon Nanotubes
}

\author{
Takahiro Mori ${ }^{1, *}$, Shunsuke Sato ${ }^{1,2}$, Kazuo Omura ${ }^{1,3}$, Shota Yajima ${ }^{1,3}$, \\ Yasuhiro Tsuruoka ${ }^{4}$, Katsumi Uchida ${ }^{3}$, Yohji Achiba ${ }^{4}$, \\ Hirofumi Yajima ${ }^{3}$ and Koji Ishibashi ${ }^{1}$ \\ 'Advanced Device Laboratory, RIKEN (The Institute of Physical and Chemical Research), \\ 2-1 Hirosawa, Wako, Saitama 351-0198, Japan \\ ${ }^{2}$ Department of Physics, Tokyo University of Science, \\ 1-3 Kagurazaka, Shinjuku-ku, Tokyo 162-8601, Japan \\ ${ }^{3}$ Department of Applied Chemistry, Tokyo University of Science, \\ 1-3 Kagurazaka, Shinjuku-ku, Tokyo 162-8601, Japan \\ ${ }^{4}$ Department of Chemistry, Tokyo Metropolitan University, \\ 1-1 Minami-Osawa, Hachioji, Tokyo 192-0397, Japan
}

(Received February 28, 2009; accepted June 1, 2009)

Key words: carbon nanotube, single-electron transistor, ion irradiation, surfactant

Single-electron transistors (SETs) are one of the possible devices for use as transducers for highly sensitive sensors that can detect small charges. We describe two approaches to fabricating high-temperature-operable single-walled carbon nanotube (SWCNT) SETs, which can operate above the temperature of liquid nitrogen, with a mass-production-adaptable process. One approach involves a SET with SWCNTs dispersed in a carboxymethylcellulose (CMC)/water suspension, which can improve the SWCNT/metal interface properties by increasing the barrier height, so that Coulomb oscillations can be observed up to $80 \mathrm{~K}$. The other approach involves a SET with a segmented Coulomb island between two higher resistance parts in an individual SWCNT, which were induced by Ar ion irradiation. This SET has been operated at up to $100 \mathrm{~K}$, and the operation temperature could be increased by the improvement in the device structure.

${ }^{*}$ Corresponding author: e-mail: tmori@riken.jp 


\section{Introduction}

The detection of small charges is one of the key issues in achieving higher sensitivity of sensors such as biosensors. Now, nanomaterial-based field-effect transistors (FETs) are widely used to improve the sensitivity of biosensors ${ }^{(1,2)}$ by improving the transconductance of FETs. Considering the detection of small charges, we can easily speculate on the great potential of single-electron transistors (SETs) as transducers of future sensors. SETs operate with a current of single-electron order on the basis of the Coulomb blockade effect.(3) Thus, SETs are also promising candidates for use as fundamental components that can act as an alternative to FETs for future electronics, because of the expectation of their use in low-power-consumption technologies. ${ }^{(4)}$

To improve the performance of SETs, single-walled carbon nanotubes (SWCNTs) are one of the natural choices as a building block of SETs because of their extremely small structure with diameters of $\sim 1 \mathrm{~nm}$. ${ }^{(3)}$ An important issue in the SET research field is the operation temperature, because it is difficult to achieve room-temperature (RT) operation of SETs. There are two device parameters that determine the operation temperature, one is the charging energy for a single electron $E_{\mathrm{C}}=e^{2} / C_{\Sigma}$, where $C_{\Sigma}$ is the self-capacitance of the Coulomb island, and the other is the tunnel barrier height $\varphi_{\mathrm{B}}$ to confine electrons in the island. The simplest device structure of a SWCNT-SET contains two metallic contacts (source and drain contacts) deposited on an individual SWCNT and the SWCNT between the contacts forms a Coulomb island, where tunnel barriers are formed at the SWCNT/metal interfaces. ${ }^{(3)}$ This device operates up to approximately $20 \mathrm{~K}$, with typical values of the parameters, which are an $E_{\mathrm{C}}$ of $10-20 \mathrm{meV}$ and a $\varphi_{\mathrm{B}}$ of about $10 \mathrm{meV}{ }^{(5)}$ In this device, the self-capacitance $C_{\Sigma}$, which is the sum of the gate capacitance and the barrier capacitances, is dominated by the barrier capacitances. Here, we can easily determine that the barrier properties play an important role in achieving the RT operation of the SWCNT-SET. By determining how to form the barriers, RT operations of the SWCNT-SET have been achieved and are reported in some papers. ${ }^{(6,7)}$

In this paper, we describe two approaches to improving the temperature characteristics of the SWCNT-SET. One approach involves the modification of the SWCNT/metal interface barriers using the simplest device structure mentioned above. The effect of the adsorbed molecules or atoms on the properties of the SWCNT/metal interface has been mentioned in some papers. ${ }^{(8-10)}$ In the fabrication process of the SWCNT-SET by the dispersion method, we must use a dispersant to obtain individual SWCNTs, and adsorbed dispersants affect the SWCNT/metal interface properties. Carboxymethylcellulose (CMC) is our choice as a dispersant, and it modified the interface properties to obtain a higher $\varphi_{\mathrm{B}}$ of approximately $20 \mathrm{meV}$, which resulted in an operation temperature of the finished SWCNT-SETs of up to $80 \mathrm{~K}$. The other approach involves barrier formation by lowenergy Ar ion irradiation. Individual SWCNTs were partly irradiated through the two openings to induce two higher resistance parts. The irradiated parts act as the tunnel barriers and the segment between the irradiated parts forms the Coulomb island. We demonstrate SET operation up to $100 \mathrm{~K}$ in this study, but the operation temperature could increase with modification of the device structure. 


\section{Dispersant Effect on Barrier Height}

To fabricate SWCNT-SETs by the dispersion method, surfactants such as Triton X-100 or sodium dodecylsulfate (SDS) are commonly used as a dispersant to produce an SWCNT suspension in which individual SWCNTs are dispersed. The SWCNT-SET with the simplest device structure, fabricated with a Triton X-100/water suspension was operated up to $20 \mathrm{~K}$ as previously reported.(5) $\mathrm{CMC}$ is a polymer, a derivative of cellulose. It can work as a dispersant similarly to surfactants, as described in ref. 11. In both cases we used the simplest device structure of the SWCNT-SETs with a back gate. The details of the device fabrication process are given in ref. 12 .

The differential conductance mapping of the SWCNT-SET fabricated with the $\mathrm{CMC} /$ water suspension is shown in Fig. 1. The series of white diamond shapes are socalled Coulomb diamonds with no flowing current, which are a standard feature of the SET. The lines outside the diamonds indicate the effect of the zero-dimensional confined state, which means that the Coulomb island is a quantum dot. The roughly estimated device parameters from the diamonds are a charging energy of $8 \mathrm{meV}$, an energy splitting of the zero-dimensional states $\Delta E$ of $2 \mathrm{meV}$, and a $C_{\mathrm{G}} / C_{\Sigma}$ ratio of 0.1 . The $C_{\mathrm{G}} / C_{\Sigma}$ ratio indicates that the barrier capacitances are dominant in the $C_{\Sigma}$.

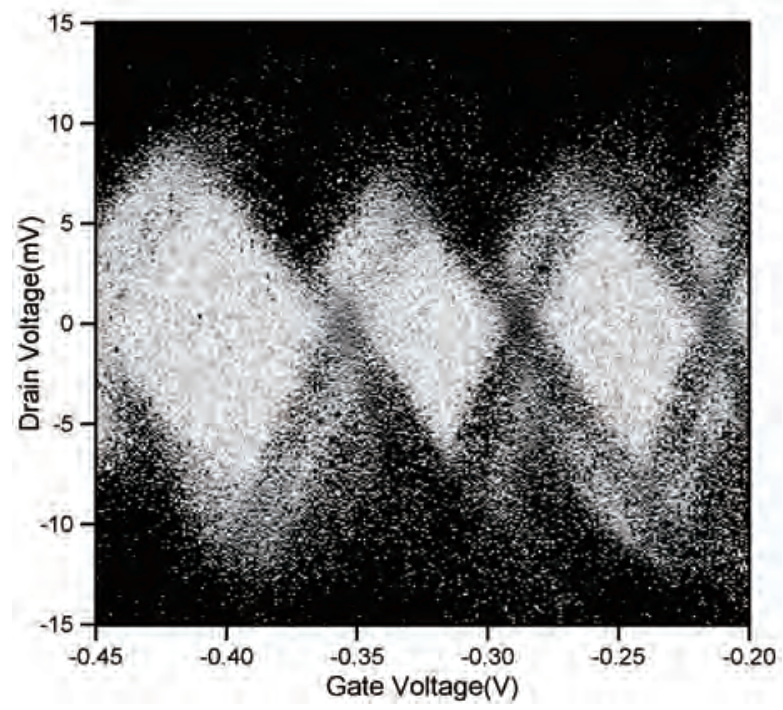

Fig. 1. Differential conductance mapping as a function of the drain and gate voltages. The measurement temperature was $1.5 \mathrm{~K}$. The white areas indicate zero conductance. Coulomb diamonds can be clearly observed with the zero-dimensional confined state lines. 
The temperature dependence of the Coulomb oscillations is shown in Fig. 2. Regular Coulomb oscillations were observed up to $80 \mathrm{~K}$, which means that the SET operates as high as $80 \mathrm{~K}$. This operation temperature was much higher than that of the SET fabricated with the Triton X-100/water suspension; however, there is no notable difference in the charging energy between these two devices. The factor that determines the operation temperature difference is $\varphi_{\mathrm{B}}$. The $\varphi_{\mathrm{B}}$ of the SET fabricated with the Triton X-100/water suspension was typically less than $10 \mathrm{meV}$, in contrast to the $\varphi_{\mathrm{B}}$ of approximately $20 \mathrm{meV}$ of the SET fabricated with the $\mathrm{CMC} /$ water suspension, as estimated from Fig. 3.

The improvement in the temperature characteristics resulted from the dispersant difference, which suggests that adsorbed dispersant molecules on the SWCNT modify the SWCNT/metal interface properties. We can easily speculate that the moleculeinduced dipoles change the surface potential of the contact metal at semiconductor/metal interfaces with the adsorbed organic molecules at the interface. ${ }^{(13,14)}$

\section{Barrier Formation with Ar Ion Irradiation Technique}

A schematic of the induction of Ar-ion-irradiated parts in an individual SWCNT is shown in Fig. 4. An individual SWCNT with two electrodes was covered with a protection resist and two openings for $\mathrm{Ar}$ ion irradiation were fabricated by electron beam lithography. The protected regions of the SWCNT had no defects induced by the Ar ion irradiation, but the opened regions exhibited induced defects and had higher resistance than the protected regions. In this paper, we describe the results achieved with a device in which the gap between electrodes was about $1 \mu \mathrm{m}$, the width of the openings was about $50 \mathrm{~nm}$, and the gap between the openings was about $300 \mathrm{~nm}$. The details of the fabrication procedure are given in ref. 15 .

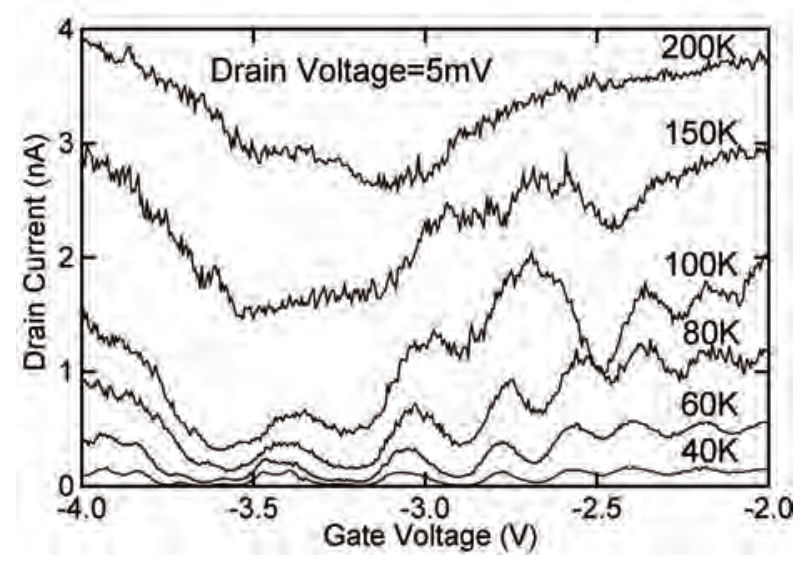

Fig. 2. Gate characteristics at 40-200 K. Coulomb oscillations can be clearly observed up to $80 \mathrm{~K}$. 


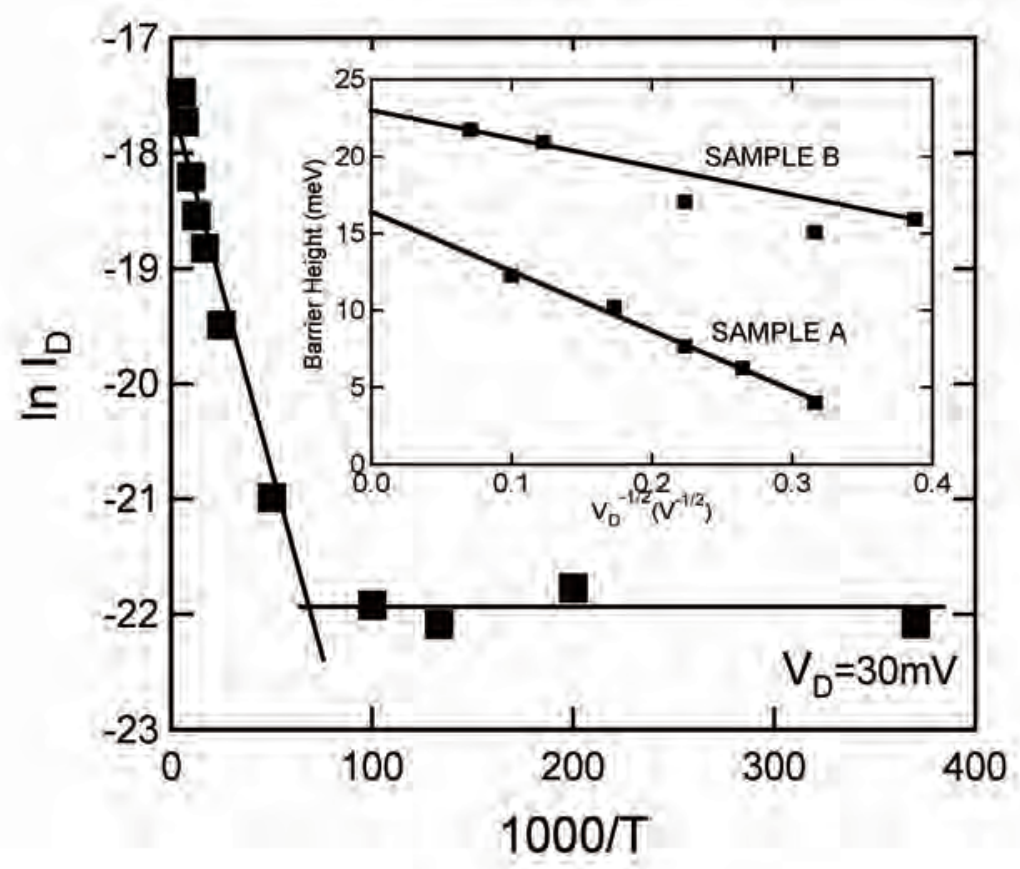

Fig. 3. Temperature dependence of the drain current with $30 \mathrm{mV}$ of drain voltage. The barrier height was estimated from the slope of the high-temperature side of the plot. The inset shows the drain voltage dependence of the barrier height.

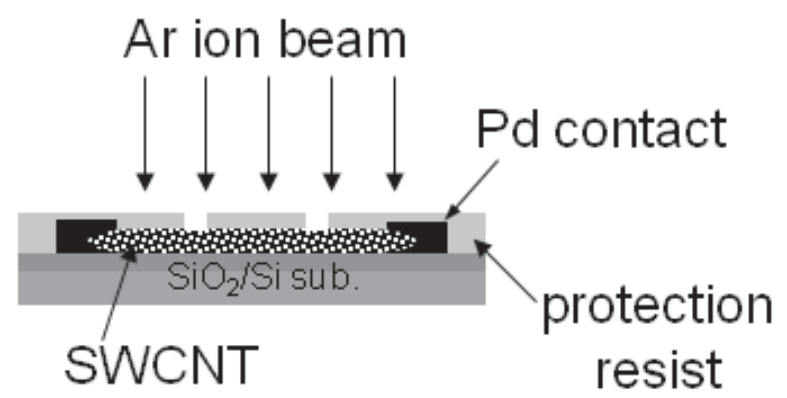

Fig. 4. Schematic cross-sectional image of the Ar-ion-irradiation process. The device was covered with protection resist and two openings were fabricated by electron beam lithography. 
The SETs fabricated by this method were successfully operated, and the temperature dependence of the Coulomb oscillations is shown in Fig. 5. Regular Coulomb oscillations were observed up to $100 \mathrm{~K}$, with a drain voltage of $30 \mathrm{mV}$. The barriers were successfully formed by low-energy Ar ion irradiation. In our device formation process, we should consider the electron irradiation effect on SWCNTs, as reported in ref. 16, because we used electron lithography to form the openings. However, no notable changes in the device properties were observed, such as a reduction in resistance or gate modulation. This may be because of a discrepancy in the electron absorption efficiency caused by the difference in electron acceleration voltages used in the experiments.

The notable feature of this device was the small barrier capacitance. The estimated sum of the two barrier capacitances was about $0.8 \mathrm{aF}$, and the gate capacitance dominated the total capacitance. Thus, we can expect much higher SET operation with the reduction in the gate capacitance, which can be achieved with the reduction in the Coulomb island size determined by electron beam lithography.

\section{Summary}

We described two approaches to fabricate SWCNT-SETs that can operate at higher temperatures. One involves barrier height modification using the polymer dispersant, carboxymethylcellulose. The fabricated SET operated up to $80 \mathrm{~K}$ with a barrier height of about $20 \mathrm{meV}$. The other involves barrier formation using Ar ion irradiation. The fabricated SET operated up to $100 \mathrm{~K}$ with small barrier capacitances of about $0.4 \mathrm{aF}$.

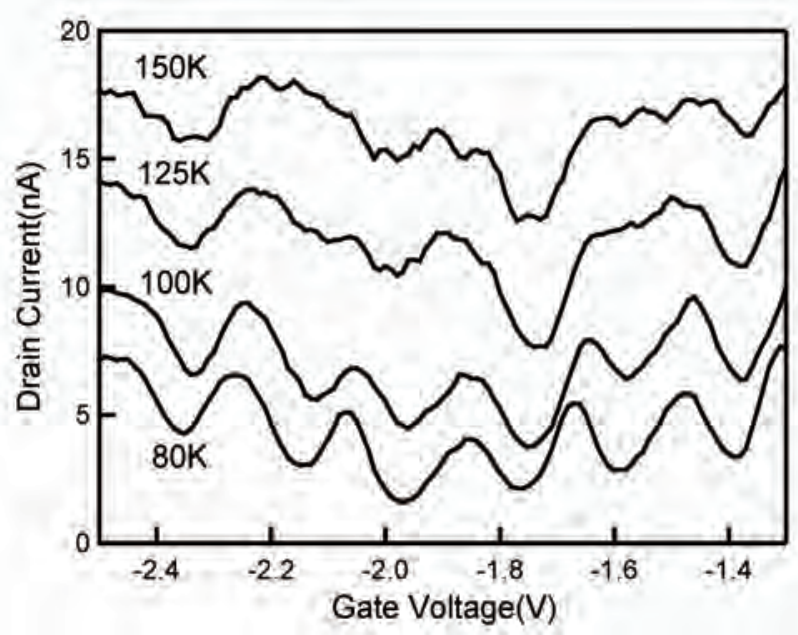

Fig. 5. Gate characteristics at $80-150 \mathrm{~K}$ with a drain voltage of $30 \mathrm{mV}$. Coulomb oscillations can be observed up to $100 \mathrm{~K}$. 


\section{Acknowledgement}

This work was partially supported by a KAKENHI, Grant-in-Aid for Young Scientists, No. 200710110, from MEXT.

\section{References}

1 G. Zheng, F. Patolsky, Y. Cui, W. U. Wang and C. M. Lieber: Nat. Biotechnol. 23 (2005) 1294.

2 K. Maehashi, T. Katsura, K. Kerman, Y. Takamura, K. Matsumoto and E. Tamiya: Anal. Chem. 79 (2007) 782.

3 K. Ishibashi, S. Moriyama and T. Fuse: IEICE Trans. Electron. E87-C (2004) 1799.

4 International Technology Roadmap for Semiconductors: http://www.itrs.net/.

5 D. Tsuya, M. Suzuki, Y. Aoyagi and K. Ishibashi: Jpn. J. Appl. Phys., Part 144 (2005) 1588.

6 H. Postma, T. Teppen, Z. Yao, M. Grifoni and C. Dekker: Science 293 (2001) 76.

7 K. Maehashi, H. Ozaki, Y. Ohno, K. Inoue, K. Matsumoto, S. Seki and S. Tagawa: Appl. Phys. Lett. 90 (2007) 023103.

8 S. Heinze, J. Tersoff, R. Martel, V. Derycke, J. Appenzeller and Ph. Avouris: Phys. Rev. Lett. 89 (2002) 106801.

9 X. Cui, M. Freitag, R. Martel, L. Brus and P. Avouris: Nano. Lett. 3 (2003) 783.

10 J. Li, Q. Zhang, H. Li and M. B. Chan-Park: Nanotechnology 17 (2006) 668.

11 T. Takahashi, K. Tsunoda, H. Yajima and T. Ishii: Jpn. J. Appl. Phys., Part 143 (2004) 3636.

12 T. Mori, K. Omura, S. Sato, M. Suzuki, K. Uchida, H. Yajima and K. Ishibashi: Appl. Phys. Lett. 91 (2007) 263511.

13 R. T. Tung: Phys. Rev. B 64 (2001) 205310.

14 H. Ishii, K. Sugiyama, E. Ito and K. Seki: Adv. Mater. (Weinheim, Ger.) 11 (1999) 605.

15 T. Mori, S. Sato, K. Omura, S. Yajima, Y. Tsuruoka, Y. Achiba and K. Ishibashi: J. Vac. Sci. Technol., B (in press).

16 T. Suzuki and Y. Kobayashi: Jpn. J. Appl. Phys., Part 244 (2005) L1498. 